\title{
Study of Oxygen Distortions in Titanate - Manganite Interfaces by Aberration Corrected STEM-EELS
}

\author{
Gabriel Sanchez-Santolino ${ }^{1,2}$, Javier Tornos ${ }^{1}$, Mariona Cabero ${ }^{1,2}$, Maria Varela ${ }^{3,1,2}$ Javier \\ Garcia-Barriocanal ${ }^{1}$, Carlos Leon ${ }^{1}$, Stephen J. Pennycook ${ }^{4}$ and Jacobo Santamaría ${ }^{1}$ \\ 1. GFMC, Dept. de Fisica Aplicada III, Universidad Complutense de Madrid, 28040 Madrid, Spain \\ 2. Instituto Pluridisciplinar, Universidad Complutense de Madrid, 28040 Madrid, Spain \\ 3. Materials Science and Technology Div., Oak Ridge National Laboratory, Oak Ridge, TN 37831, USA \\ 4. Dept. of Materials Science and Engineering, University of Tennessee, Knoxville, TN 37996, USA
}

Transition metal oxides constitute a most interesting family of materials thanks to the interplay between structure, electronic and orbital degrees of freedom, which are related to collective phenomena like magnetism, ferroelectricity, superconductivity, electron transfer, etc. Interfaces between complex oxide materials provide a promising scenario for novel physical phenomena to arise. The crystal and electronic structures of these interfaces determine their physical behaviors [1]. In particular, distortions from the perfect cubic perovskite structure, such as deformations in the $\mathrm{BO}_{6}$ oxygen octahedron around the cations and collective tilts and displacements of the oxygen sub-lattice, play an important role in the electronic properties [2]. In order to harness the macroscopic properties of oxide interfaces, techniques capable of real space simultaneous characterization of these systems with atomic resolution are needed. Aberration-corrected scanning transmission electron microscopy (STEM) combined with electron energy loss spectroscopy (EELS) is a most powerful tool to study the structure, chemistry and electronic properties of these oxide interfaces down to the atomic scale and in real space. The high spatial resolution achieved allows the acquisition of annular bright field (ABF) images, sensitive to light atoms like oxygen, and atomic resolution spectrum images [3].

Here, we use both techniques to study the oxygen distortions in different manganite/titanate interfaces. First, we will discuss the interface between the Mott insulator $\mathrm{LaMnO}_{3}$ (LMO) and the band insulator $\mathrm{SrTiO}_{3}$ (STO) in superlattices with different thickness ratios. In this system, the macroscopic physical properties can be tuned by adjusting the relative LMO/STO layer thicknesses, resulting in different behaviors from a ferromagnetic metal to a non-magnetic insulator [4]. The LMO/STO thickness ratio can be used to tune the degree of mismatch strain in the system and hence it has strong effects on the interface structure. We have measured the strain through the samples and also the octahedral geometry (by imaging the oxygen positions) using atomic resolution energy-loss spectrum images, as shown in Figure 1. We find significant changes in the oxygen octahedral tilts both in STO and LMO when the thickness ratio between the manganite and the titanate is varied. These structural variations could be related to differences in the transport and magnetic properties of this system.

We have also studied the multiferroic system between the ferromagnetic $\mathrm{La}_{0.7} \mathrm{Sr}_{0.3} \mathrm{MnO}_{3}$ (LSMO) and the ferroelectric $\mathrm{BaTiO}_{3}$ (BTO). In this case, the strain across the interface can have profound implications on the ferroelectricity in BTO, as well. In order to understand the system properties, monitoring the atomic positions across the interfaces is a must. We can measure the atomic column positions directly on the ABF images obtained by STEM, as shown in Figure 2, which allows the calculation of relative displacements between the oxygen and titanium atoms, and hence, the study of polarization at a local scale. We observe polarization gradients between the manganite and the ferroelectric, possibly associated with strain gradients, and the presence of oxygen vacancies that may 
help to accommodate these lattice distortions. Finding subtle aspects of the material structural, electronic and chemical properties could explain the relationship between the microscopic and macroscopic physical behaviors of these systems and help in the designing of devices with novel functionalities and in the development of novel materials.

\section{References:}

[1] Reyren, N. et al., Science 317 (2007), 1196.

[2] Jia, C. L. et al., Physical Review B, 79 (2009), 081405.

[3] Varela, M. et al., Physical Review B, 79 (2009), 085117.

[4] Garcia-Barriocanal, J. et al., Advanced Materials 22, (2010), 627.

[5] Sanchez-Santolino, G. et al., Microscopy and Microanalysis, (2014) submitted.

[6] The authors thank Masashi Watanabe for the Digital Micrograph PCA plug-in, Andrew Lupini for the atom mapping scripts and J. Luck for help with the specimen preparation. Research at ORNL was supported by the U.S. Department of Energy, Basic Energy Sciences, Materials Sciences and Engineering. Research at UCM was supported by Spanish MICINN through grants MAT2011-27470C02 and Consolider Ingenio 2010-CSD2009-00013 (Imagine), by CAM through grant S2009/MAT1756 (Phama) and by the ERC Starting Investigator Grant \#239739 STEMOX.

a)

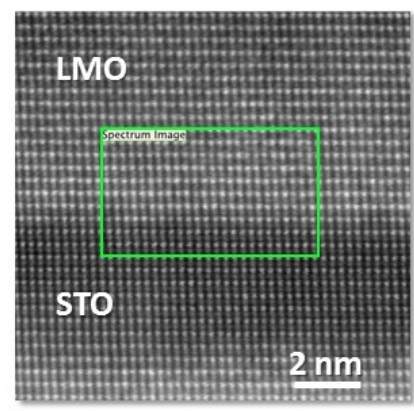

b)

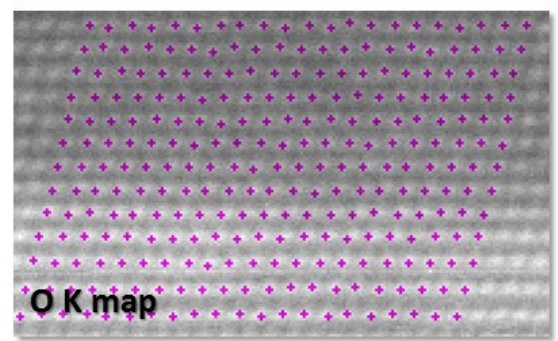

Figure 1. (a) High angle annular dark field image obtained in a Nion UltraSTEM100 of an interface between LMO and STO, down the (110) orientation. (b) EELS oxygen $K$ edge integrated signal map from the area marked in (a). The pink crosses mark the oxygen atoms positions. Some spatial drift is visible. Adapted from reference [5].

a)

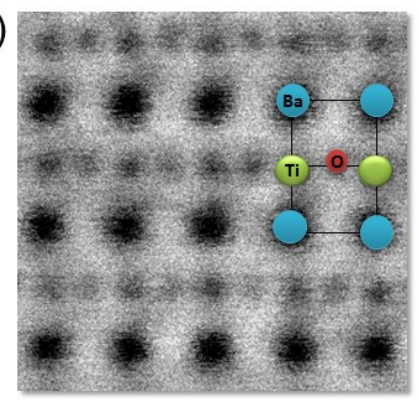

b)

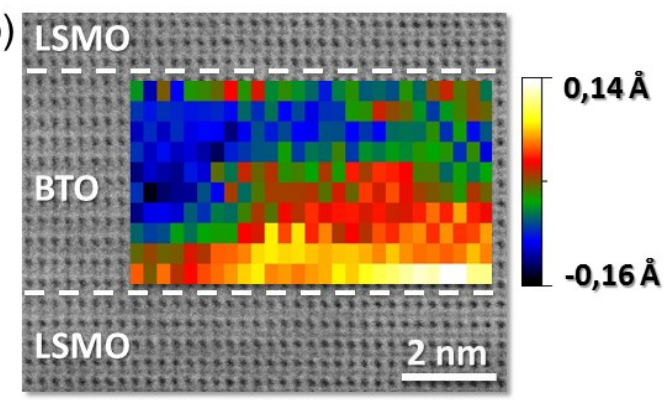

Figure 2. (a) Annular bright field image of BTO down the pseudocubic (110) axis obtained in a Nion UltraSTEM200 of BTO with a sketch of the unit cell structure (b) ABF image of a LSMO/BTO superlattice with a titanium-oxygen relative displacements map superimposed. Each pixel in the map corresponds to one unit cell. 